\title{
Gynecologic Pathology: Practical Issues and Updates
}

Editor

BROOKE E. HOWITT

\section{SURGICAL PATHOLOGY CLINICS}

www.surgpath.theclinics.com

Consulting Editor

JASON L. HORNICK

June 2019 • Volume 12 - Number 2 